\title{
Free-form Flexible Lithium-Ion Microbattery
}

\author{
Arwa T. Kutbee, Mohamed T. Ghoneim, Student Member, IEEE, Sally M. Ahmad, and Muhammad \\ M. Hussain, Senior Member, IEEE
}

\begin{abstract}
Wearable electronics need miniaturized, safe and flexible power sources. Lithium ion battery is a strong candidate as high performance flexible battery. The development of flexible materials for battery electrodes suffers from the limited material choices. In this work, we present integration strategy to rationally design materials and processes to report flexible inorganic lithiumion microbattery with no restrictions on the materials used. The battery shows an enhanced normalized capacity of $147 \mu \mathrm{Ah} / \mathrm{cm}^{2}$ when bent.
\end{abstract}

Index Terms - Thin films, lithium-ion, flexible, microbattery.

\section{INTRODUCTION}

$\mathrm{F}$ LEXIBLE batteries are sought for the new era of wearables and Internet of Everything (IoE) electronic systems. We envision a world where every things will be smart with embedded sensors, electronics and power sources for data exchange and communication through the World Wide Web. To realize such a world, we have expanded the application of electronics toward human, animal, plants, etc. However, conventional ionic and electronic devices are rigid and nonconformal to the human body or skin, restricting their usage in a truly wearable platform. Recently, many researchers have reported on the fabrication of various flexible devices such as flexible LEDs [1], thermoelectric generators [2, 3], photovoltaics [4], and logic devices [5]. Additionally, flexible super capacitors [6-8], and batteries [9, 10] have been developed as a main power source to sustain the usage of IoE devices. Among these, lithium ion batteries (LIBs) offer large cycle life and high energy density, making them attractive for IoE and wearable electronics. However, commercialized LIBs of various form factors such as coin, cylindrical, prismatic and pouch cells are not suitable for the next generation flexible batteries. Additionally, specific IoE applications targeted toward implantable medical devices impose other constrains on the LIB technology such as non-toxicity, size miniaturization and integration with other circuit components.

Recent advances in research are trying to push the form factor of lithium-ion technology towards free-form: flexible, thin, light weight, and small batteries. Two key strategies are

All copyrights have been transferred by the authors to IEEE and are reserved by IEEE. The author would like to thank Cymbet and Micross Corporation for bare die supply. The author appreciates the generous baseline funding from King Abdullah University of Science and Technology. The author also thanks the support from the staffs in the KAUST Advanced Nanofabrication Facilities (KANF) at the King Abdullah University of Science and Technology. demonstrated in the literature [11-13]. The first strategy focuses on developing flexible materials for all electrochemical cell components comprising: current collectors, electrolyte, and electrodes. Flexible current collectors are necessary components to maintain structural stability and conduction with active materials such as: carbon nanotube (CNT) paper [14], graphene fiber [15], conductive paper (cellulous) [16], and textiles based on conductive carbon cloth [17]. On the other hand, electrolytes based on solid state [18], gel polymer [19], and plastic crystal materials [20] were developed to replace liquid electrolytes that are prone to chemical leakage. Another development is targeted toward active materials, which are considered as the bottlenecks for high performance LIBs. These can be made from slurry-type conventional transition metal oxides, conductive carbon, or nano-structured materials such as CNT [21] and graphene [22]. However, composites of slurrytype metal oxides and nanostructured materials mixed homogenously promise higher capacity with improved flexibility [23].

Materials investigation present a promising route for flexible batteries, but their widespread deployment for large-scale applications is limited to the synthesizing methods which are only restricted to a few pairs of anode and cathode materials with specific structures. Additionally, their performance is not sufficient, requiring deeper understanding of the underlined chemistries of the newly developed materials.

A second strategy focuses on inherently non-flexible materials of reduced thicknesses [24-26]. For a specific bending radius, the material's fracture strain induced by bending can be linearly decreased with thickness. Thin film deposition combined with microfabrication techniques have been implemented to obtain a wide range of high quality materials and pattern them into complex microstructures with sub-10 nm resolution. In the case of batteries based on thin films, solid state materials are intrinsically safe and ideal for CMOS integration. Each layer being a few microns in thickness

A. T. Kutbee, S. M Ahmad, M. T. Ghoneim, and M. M. Hussain are with the Integrated Nanotechnology Lab and Integrated Disruptive Electronic Applications (IDEA) Lab, Electrical Engineering, CEMSE Division, King Abdullah University of Science and Technology (KAUST), Thuwal 239556900, Saudi Arabia (E-mail: muhammadmustafa.hussain@kaust.edu.sa). 


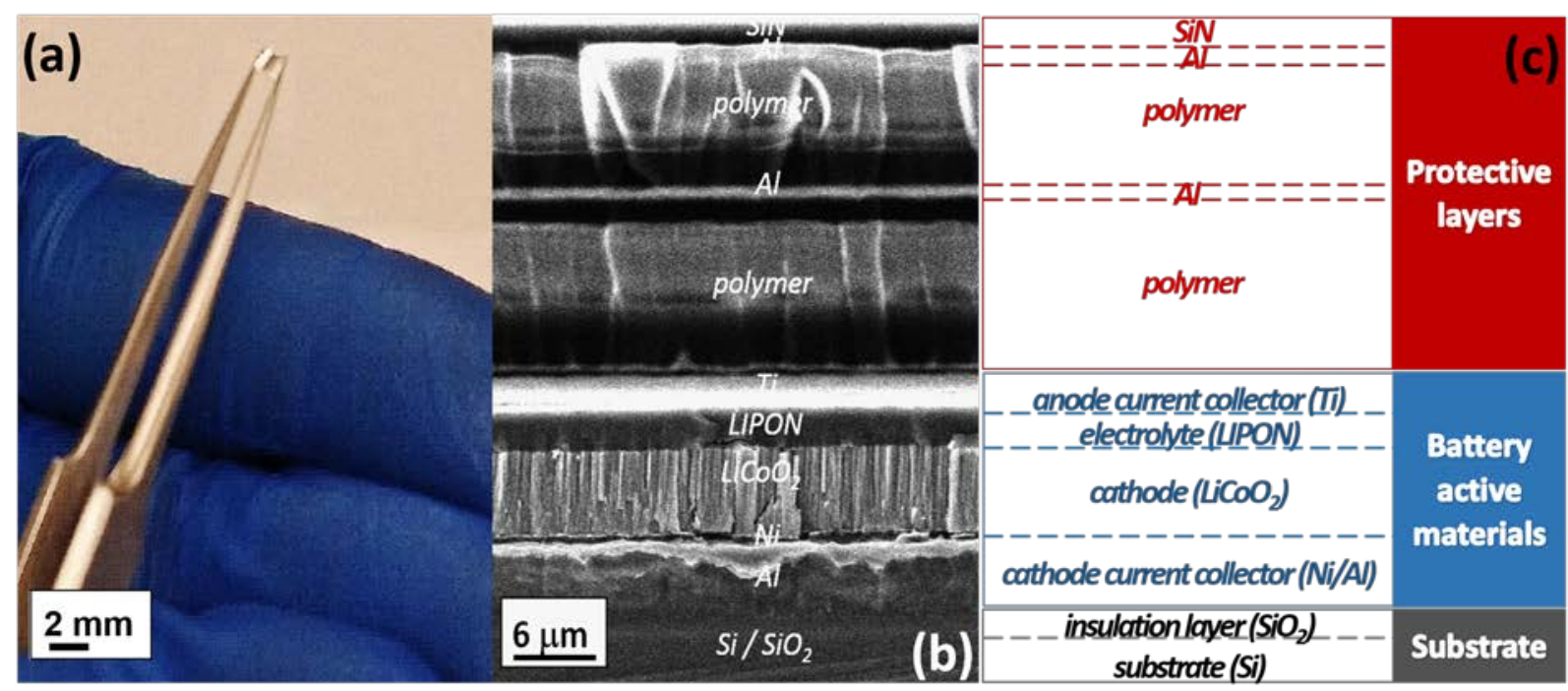

Fig. 1. (a) Optical image of a flexible LIB with a total thickness of $30 \mu \mathrm{m}$ after complete removal of the Si substrate. (b) and (c) SEM cross section image and corresponding schematic of the LIB stack prior to flexing. The LIB is built on a Si substrate with a total thickness of $175 \mu \mathrm{m}$. The thickness of the flexible LIB of $30 \mu \mathrm{m}$ includes the total thickness of the protective layers and active materials of the LIB without the Si substrate.

decreases the diffusion length needed for charge transport.

Thin film batteries are prepared on conventional monocrystalline Silicon (Si) to maintain its mechanical integrity. Nevertheless, the usage of the rigid Si substrate limits the potential of thin film LIB in flexible electronics. Efforts are dedicated to replace conventional rigid substrates with buckled polymers in order to obtain a flexible metallic collector [27].

However, plastics are incompatible with high thermal budget processes such as annealing. Specifically, in thin film batteries, metal oxide films of high storage capacity require annealing temperatures above $600{ }^{\circ} \mathrm{C}$. Therefore, the usage of thermally stable sacrificial substrate and transferring the devices onto a final destination polymeric matrix has been developed using either a device last approach or a device first approach.

In a device last approach, Rogers et al. fabricated a stretchable battery module with multiple pouch cells [28]. Although such a cell is a not considered as a thin film battery, it is prepared by microfabrication techniques on Polymethylmethacrylate (PMMA) coated Si sacrificial substrates for the patterning and transferring of thin film current collector with two levels of serpentine structures for fractal design enabled stretching. A soft lithographic type casting method was done separately for the active slurry-based materials. The pouch cell was assembled on a final destination polymeric matrix in a process known as transfer printing. The stretchable battery module was able to achieve $>300 \%$ stretchability. However, the complexity associated with the transfer steps and aligning the device layers with respect to each other limits the miniaturization of the LIB.

On the other hand, a device first approach to develop a flexible battery from an all solid state thin film battery was introduced by Kim et al. [29]. The processes exploits the exfoliation of a sacrificial mica substrate to release the already deposited battery stack from the underneath substrate. The problem associated with exfoliation is that it has low yield and is time consuming for large-scale applications. Additionally, the usage of a reactive metallic lithium as an anode is disadvantageous because of the complexity and safety issues associated with its preparation and usage.

In this work we show an integration strategy for a flexible thin film battery with an anode which serves as same as the current collector, referred to as "'Lithium-free"' LIB [30]. The thin film stack [31] can withstand soldering temperatures (200 ${ }^{\circ} \mathrm{C}$ ) and avoids the lithium metal dangers. In the context of our work, we develop a free-form flexible version of this lithiumfree LIB. Such a free-form flexible lithium-free LIB is attractive because it is non-cytotoxic and safe to be implemented in medical applications (including in-vivo and invitro) for IoE and wearable electronics [32]. Our device first approach with the complete removal of the sacrificial Si (100) substrate and a newly developed transfer scheme gives a flexible LIB with small size. Additionally, our approach for a flexible battery is advantageous owing to its compatibility with complementary metal oxide semiconductor (CMOS) etching processes, high yield production and ease of handling of fragile flexible samples. The battery showed an enhanced normalized capacity of $146 \mu \mathrm{Ah} / \mathrm{cm}^{2}$ at a rate $130 \mu \mathrm{A} / \mathrm{cm}^{2}$ (1C).

\section{EXPERIMENTAL}

A commercial LIB was used to demonstrate the versatility of our approach and its direct application to the available solidstate battery market. Using our device first approach, we convert thin film all-solid state LIB (EnerChip CBC 005 bare die, Cymbet, USA) with an area of $2.25 \mathrm{~mm} \times 1.7 \mathrm{~mm}$ and an overall thickness of $175 \mu \mathrm{m}$ into a flexible free-form form LIB with a thickness of $30 \mu \mathrm{m}$ as shown in Fig. 1(a). The SEM cross section image in Fig. 1(b) obtained using FEI Quanta ${ }^{\mathrm{TM}}$ 3D equipped with an energy dispersive spectroscopy (EDS) detector for elemental identification, shows the battery stack before substrate removal. The battery consists of a typical Li- 
free LIB stack with an oxidized Si substrate, an aluminum (Al) cathode current collector, a lithium cobalt oxide $\left(\mathrm{LiCoO}_{2}\right)$ cathode, a solid state lithium phosphorous oxynitride (LiPON) electrolyte, a titanium (Ti) anode current collector, and protective layers.

Unlike semiconductor-based devices, the Si substrate in a thin film battery is not an active material and has no role besides supporting the battery stack during deposition. Therefore, complete removal of the substrate will allow the battery to achieve its thinnest possible state with the maximum flexibility. Our device first approach, combines the benefits of Polydimethylsiloxane (PDMS) handler substrate in Fig. 2(a) and the gentle dry etching of the backside of the Si substrate as summarized in Fig. 2(b-f). It is to be noted that the Si does not need to be mono-crystalline silicon. For low cost production we can use amorphous and poly-crystalline silicon too.

The first step is the preparation of a PDMS handler substrate. The PDMS substrate consists of two PDMS layers. For the first PDMS layer, we started with a thermally oxidized Si wafer. The wafer was sputter coated with a gold (Au) layer of $100 \mathrm{~nm}$ and a cobalt (Co) of $10 \mathrm{~nm}$ as an adhesion layer. This wafer provided necessary surface for the preparation of the PDMS handler substrate. When PDMS was poured on the Au-coated wafer, the poor adhesion properties between PDMS and Au, permitted easy retrieval of the PDMS from the wafer surface without any surface functionalization methods. The PDMS was prepared in a 10:1 mixing ratio (base to cross-linker). Bubble removal by degassing the mixture in vacuum was done to obtain a PDMS with better elasticity. The PDMS was spun coated with $500 \mathrm{rpm}$ for $30 \mathrm{~s}$, cured on a hotplate at $150^{\circ} \mathrm{C}$ for 10 minutes. The PDMS handler substrate of a thickness of $100 \mu \mathrm{m}$ was punched to $8 \mathrm{~mm}$ disks. The second layer of PDMS handler substrate was created on top of the cured PDMS disks with a thickness of $10 \mu \mathrm{m}$ by spinning at $1500 \mathrm{rpm}$ for $30 \mathrm{~s}$. After the completion of spinning, the LIB die was placed in an inverted position on top of the PDMS thin layer shown in Fig. 2(b). The non-cured PDMS matrix encapsulating the inverted die was left to cure at room temperature. In this stage, the curing at room temperature for 24 hours was done to avoid any unwanted thermal expansions of the PDMS and exposing the LIB to any temperatures that can crystalize the solid state amorphous electrolyte. This PDMS based transfer method did not need any type of glue for handling the device before or after thinning. Additionally, usage of PDMS instead of photoresists as hard mask avoided exposing the device to any solvents required to remove them, which could have damaged the polymeric protection layer in the LIB.

The second step was carried by selectively etching the Si back surface using a plasma-less Xactic Xenon difluoride $\left(\mathrm{XeF}_{2}\right)$ release etch system in Fig. 2(c). Since $\mathrm{XeF}_{2}$ dry etching is highly isotropic, the PDMS encapsulating layer acted as a hard mask, preventing any unwanted side wall etching. The samples were laid flat on top of an aluminum foil in the etching chamber. The automated $\mathrm{XeF}_{2}$ etching program consisted of multiple controlled number of cycles. Each cycle comprised a venting step for $10 \mathrm{~s}$ and an etching step for $30 \mathrm{~s}$. The etching was carried out at room temperature and a $\mathrm{XeF}_{2}$ pressure of 4 (a)
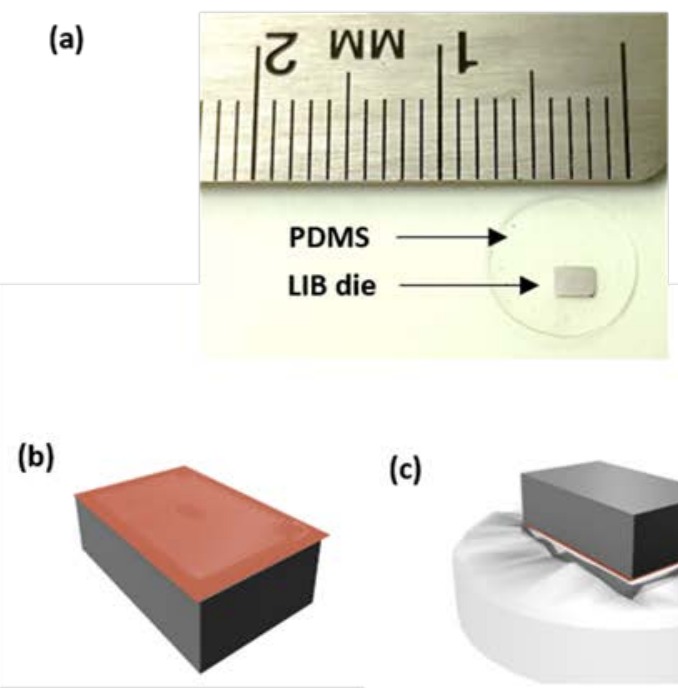

(c)
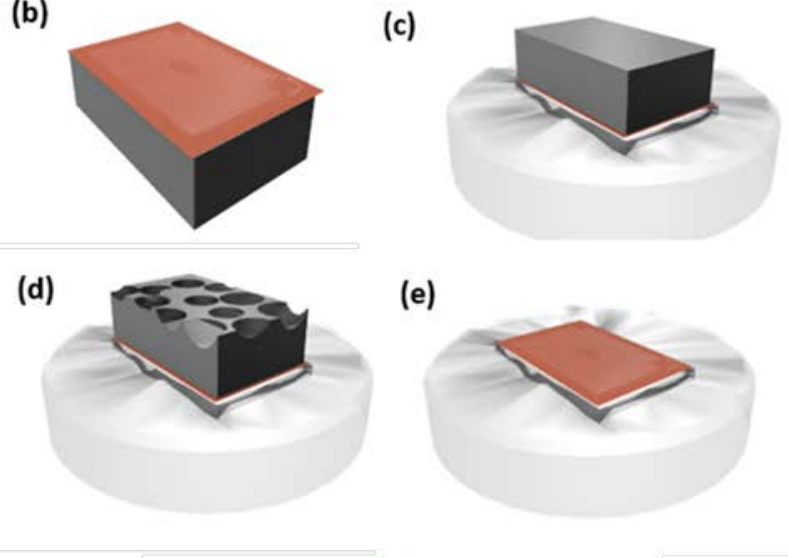

(e)

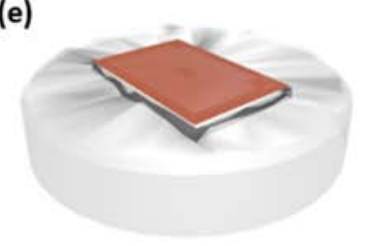

(f)

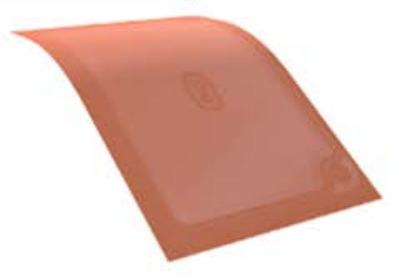

(g)

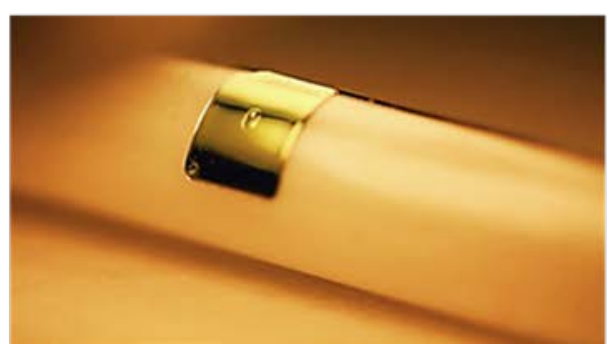

Fig. 2. (a) Optical image of a flexible battery on PDMS handler substrate; (b-f) process flow for a flexible LIB; (b) thin film LIB on a Si substrate; (c) inverted die on PDMS handle substrate. (d) $\mathrm{XeF}_{2}$ back side etch; (e) flexible LIB with back side removed completely; (f) flexible LIB after peeling from the PDMS; (g) optical micrograph of flexible battery on bending surface.

Torr. The complete consumption of the backside $\mathrm{Si}$ as shown in Fig. 2(e) was obtained with 75 cycles for a total etching time of $50 \mathrm{~min}$. The etch rate of $\mathrm{Si}$ is estimated to be $3 \mu \mathrm{m} / \mathrm{min}$. After all the etching was completed, the battery was peeled off from the fragile PDMS layer shown in Fig. 2(f). A standalone thin film robust stack was obtained with a thickness of $30 \mu \mathrm{m}$. 

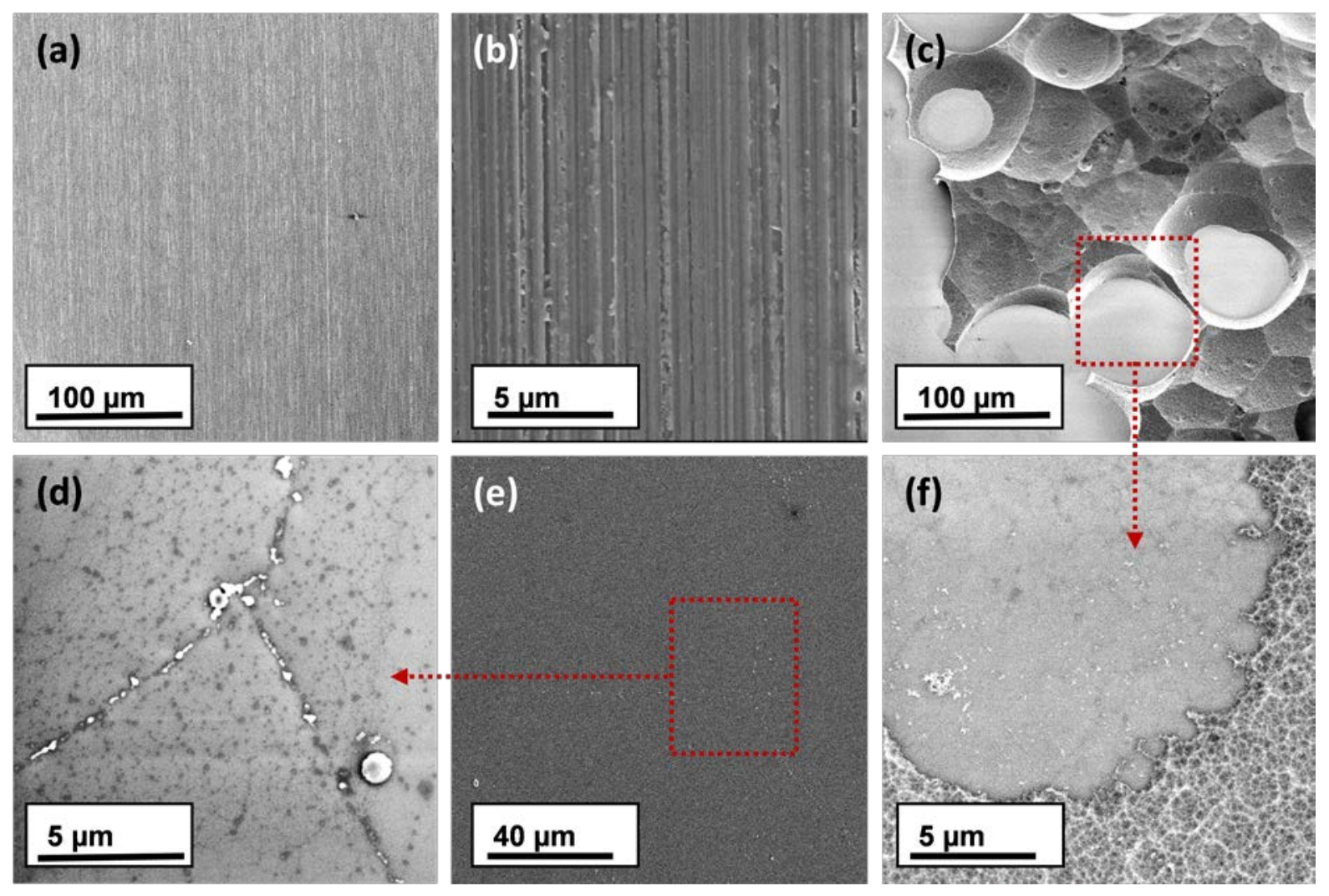

Fig. 3. SEM micrographs of surface evolution of Si substrate by $\mathrm{XeF}_{2}$ etching process of the backside of the LIB to obtain a flexible LIB. (a) and (b) Si surface before etching shows typical grinding grooves. (c) and (f) granular morphology of $\mathrm{XeF}_{2}$ etched Si surface after 30 etching cycles. (e) and (d) complete removal of Si from the backside of LIB after 75 etching cycles.

Surface morphology analysis using FEI Quanta 3D with an operating voltage of $5 \mathrm{kV}$ was obtained to characterize the $\mathrm{Si}$ surface during different etching steps and to confirm the complete removal of $\mathrm{Si}$ from the back side of the die. Reflectrometer was measured to estimate the remaining thickness of Si upon complete removal of Si. Galvanostatic testing of the battery with a pad size of $30 \mu \mathrm{m}$ was carried out using a Semi probe probe station and a Keithley (SCS) 4200 semiconductor characterization system.

An Automated battery cycling program was done to characterize the battery in the SCS 4200 . The source measure unit in the SCS 4200 can be configured as source for sourcing power and as a sink for dissipating power depending on the polarity of the voltage and current (same polarity for sourcing and opposite polarity for sinking). Two interactive test module (ITM) were created individually for battery charging and discharging. A constant current was used to charge/discharge the battery by setting a current compliance at the desired level. A voltage limit is programmed for charging up to $4.2 \mathrm{~V}$ and discharging down to $3 \mathrm{~V}$ in order to avoid overcharging/over discharging the battery, which causes unwanted structural changes and phase transformation of cathode material in the LIB, preventing it from further cyclic behavior.

\section{RESULTS AND DISCUSSION}

\section{A. Surface characterization}

SEM surface images were obtained to characterize the $\mathrm{Si}$ surface in three stages: (1) before etching, (2) after the completion of 30 etching cycles, and (3) after the completion of 75 etching cycles. In the first stage, the surface of the bare die [shown in Figs. 3(a, b)] shows a grind scratch profile with 0.2 $\mu \mathrm{m}$ wide strips. The grooving results from grinding a standard Si wafer with a $500 \mu \mathrm{m}$ into a thickness of $175 \mu \mathrm{m}$. Continued grinding below $100 \mu \mathrm{m}$ results in mechanical stresses and wafer bowing. Therefore, dry back etching via a $\mathrm{XeF}_{2}$ gas and a PDMS based transfer method is a much gentler process with a higher yield.

The surface morphology of an etched sample at 30 cycles shows a granular surface structure as a result of pitting corrosion was observed in Fig. 3(c). The pits with circular geometry has an average diameter of $50 \mu \mathrm{m}$. The Si surface roughness is ranging from a few microns to tens of microns with a porous microstructure as observed in Fig. 3(d). $\mathrm{XeF}_{2}$ etching is a known dry etchant for bulk Si micromachining. The etching mechanism of $\mathrm{Si}$ via $\mathrm{XeF}_{2}$ vapor phase molecules is the dissolution of the gas into Flourine (F) and Xenon (Xe). The fluorine atoms adsorb on the Si surface and reacts chemically with the $\mathrm{Si}$ to form a desorbing $\mathrm{SiF}_{4}$ as a gaseous molecule. The 

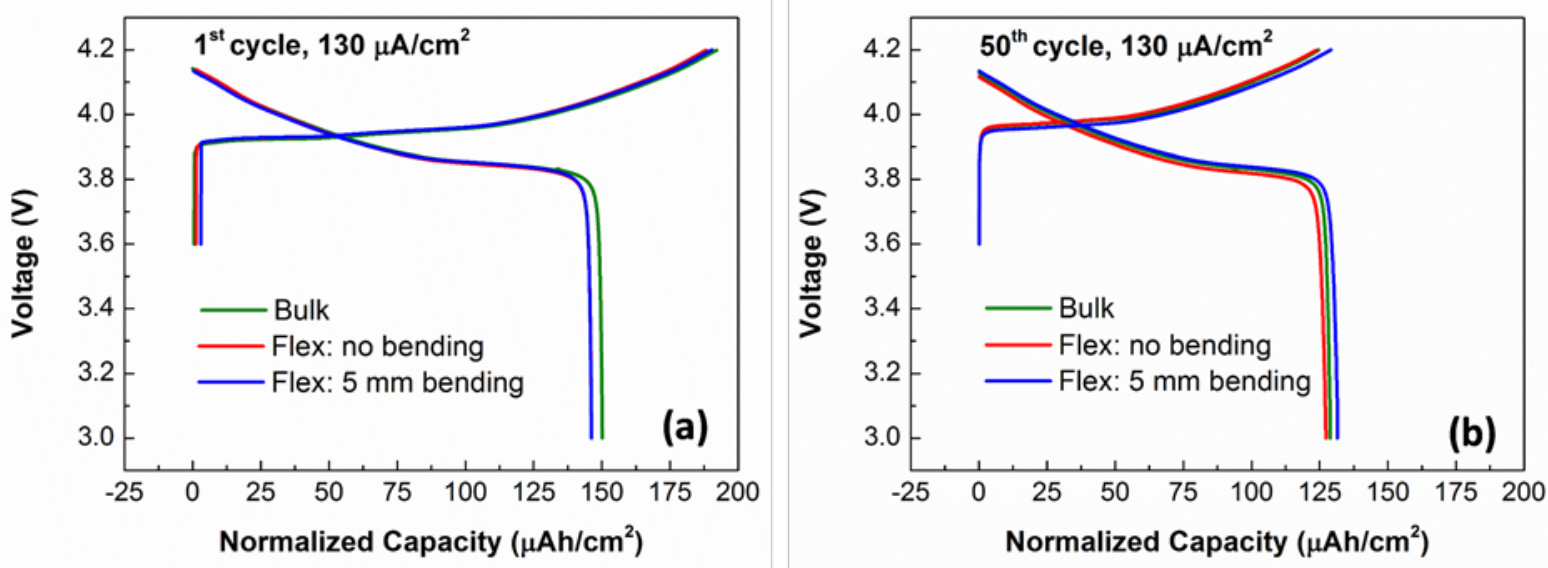

Fig. 4. Galvanostatic charge/discharge characteristics for a $1.7 \mathrm{~mm} \times 2.25 \mathrm{~mm}$ LIB cells with three states: bulk, flexible with no bending and flexible with a $5 \mathrm{~mm}$ bending radius obtained at the (a) $1^{\text {st }}$ and (b) $50^{\text {th }}$ cycles. Current rating is $130 \mu \mathrm{A} / \mathrm{cm}^{2}$ in both charge and discharge cycle.

presence of microenvironments of this chemical process on the Si surface results in the formation of pits. Regions around the pits are more immune to corrosion than the active region inside the pits.

Continued etching results in the complete removal of the surface roughness shown in Figs. 3(d, e). The Si present in the back side is consumed completely after 50 minutes of etching. Thus, SEM images confirms that the etching stops due to the presence of the $\mathrm{SiO}_{2}$ insulating layer, which is highly selective to $\mathrm{XeF}_{2}$. Reflectometer measurements of the backside confirmed the removal of the Si from the backside.

\section{B. Electrochemical characterization}

The battery characterization showed the typical potential profile for a lithium-free $\mathrm{LIB}$ with a $\mathrm{LiCoO}_{2}$ cathode. The LIB being a secondary battery is rechargeable because it relies on an intercalation mechanism for $\mathrm{Li}$ ion insertion/extraction from the $\mathrm{LiCoO}_{2}$ lattice which presents the only source for $\mathrm{Li}$ ions. On the other hand, the lithium-free LIB anode is the same as the current collector. Therefore, the battery is free from the presence of metallic lithium at the anode and is assembled in a fully discharge state. During the battery's first charge, the $\mathrm{LiCoO}_{2}$ cathode is oxidized (delithiated) into $\mathrm{Li}_{0.5} \mathrm{CoO}_{2}$, and $\mathrm{Li}+$ ions start to migrate to the anode current collector where they are reduced. As a result, $\mathrm{Li}$ is deposited on the anode current collector in a process known as "in-situ" plating. On the discharge step, the in-situ plated lithium is oxidized and $\mathrm{Li}$ ions transport back the $\mathrm{LiCoO}_{2}$ lattice. The cell voltage indicates the electrochemical potential of lithium in the cathode material with respect to a lithium reference. The capacity relates the extraction of $1 \mathrm{~mol}$ of lithium to for every $2 \mathrm{~mol}$ of the transition metal oxide.

Galvanostatic testing of the battery was carried out using a Keithley 4200 SCS. The battery cycled for 50 times with the same rating for charge and discharge of $130 \mu \mathrm{A} / \mathrm{cm}^{2}$.

In order to study the mechanical robustness of the free-form
LIB, comparison of the electrochemical performance of the LIB was done in three states: (1) Bulk state with a thin film battery on a $\mathrm{Si}$ substrate. (2) Flex state stands for a flat standalone thin film stack of the LIB with complete removal of the Si substrate. (3) Flex with $5 \mathrm{~mm}$ bending state was done by characterizing the battery on a nonconductive bended curvature with a radius of $5 \mathrm{~mm}$.

The electrochemical data for the different states at the first charge/discharge cycle [shown in Fig. 4(a)] shows a comparable behavior for all the cells with a charging capacity of $180 \mu \mathrm{Ah}$ and a discharge capacity of $147 \mu \mathrm{Ah} / \mathrm{cm}^{2}$. The discharge was completed in one hour, i.e. $1 \mathrm{C}$ rating. The discharge voltage profile for all the batteries at different states exhibit a flat voltage profile, indicating small cell dependence on the cell's state of charge in the voltage plateau from 4.2 to 3 V.

A similar voltage profile [shown in Fig. 4(b)] can be observed for the batteries after 50 charge/discharge cycles. However, an $18 \%$ discharge capacity drop is observed between the $1^{\text {st }}$ and the $50^{\text {th }}$ charge cycle, giving a drop of capacity from a value of $147 \mu \mathrm{Ah} / \mathrm{cm}^{2}$ to $127 \mu \mathrm{Ah} / \mathrm{cm}^{2}$. To understand this, cycling retention data for both charge and discharge cycles was obtained for all battery states as summarized in Figs. 5(a, b). As can be seen in Fig. 5(a) the drop of capacity is related to the drop of charge capacity after the first charge, while the discharge capacity retention stays stable after the first charge cycle shown in Fig. 5(b). The drop in charge capacity after the $1^{\text {st }}$ cycle can be attributed to the formation of solid-state interface on the electrolyte and the irreversible lithium intercalation onto the anode current collector.

Nevertheless, the free form flexible battery showed good discharge capacity retention upon cycling as observed in Fig. 5(b). It is interesting to notice that a slightly better capacity retention was observed for the flexible battery in the flexible state with $5 \mathrm{~mm}$ bending radius. Kim et al. observed an opposite 
(a)

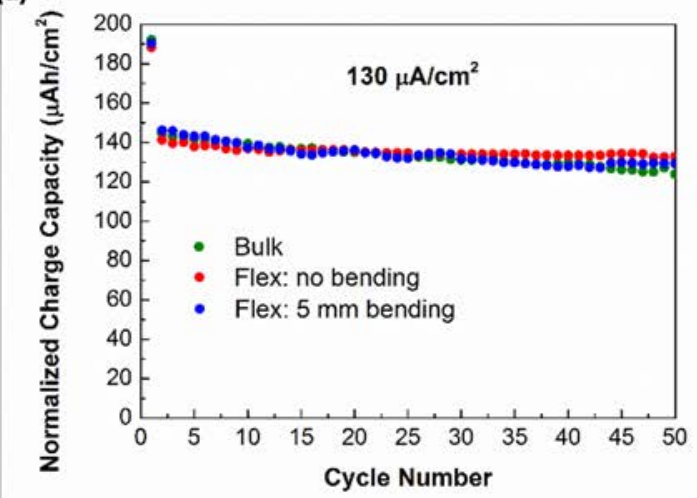

(b)

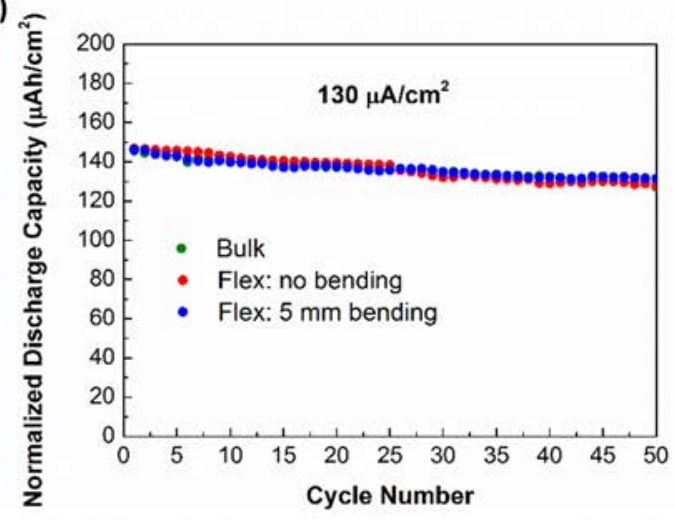

(c)

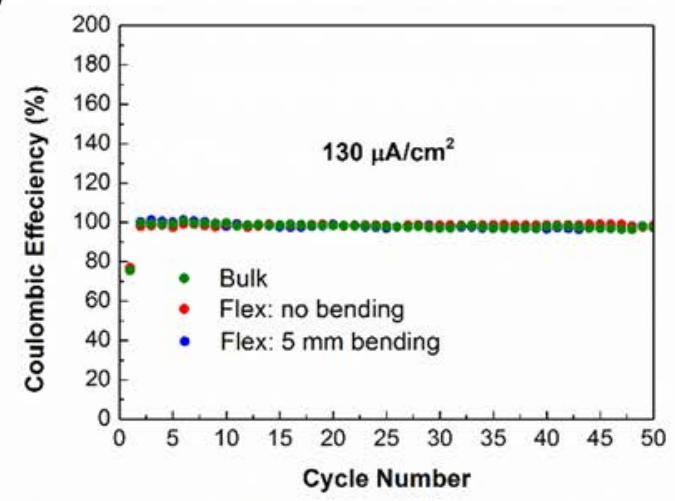

Fig. 5 Cycling stability of a LIB: (a) charging and (b) discharge capacity retention during 50 galvanostatic charge/discharge cycles. (c) Coulombic efficiency of LIB. All cells were cycled between 3 and $4.2 \mathrm{~V}$ at a constant charged/discharged current density $(=1 \mathrm{C} / 1 \mathrm{C})$.

result with an unfavorable behavior for battery retention upon cycling during bending. The unfavorable behavior was correlated to the material stress. Variation in results may be attributed to the difference in chemical composition of the stack and the utilization of PDMS encapsulation by Kim et al. [29]. Additionally, according to our results and recent findings found by density functional theory (DFT) [33], the capacity for a LIB with a $\mathrm{LiCoO}_{2}$ cathode can be enhanced upon bending if a stress is applied in a direction along the C-axis of the $\mathrm{LiCoO}_{2}$ rhombohedral lattice. Therefore, more understanding of the correlation between the effect of mechanical stress on the material properties of the $\mathrm{LiCoO}_{2}$ thin film upon bending and its direct correlation to the electrochemical behavior of the cell.

The coulombic efficiency was calculated by measuring the ratio of number of charges entering the battery to the number of charges leaving the battery. The coulombic efficiency for the batteries at different bending states was approximately $97 \%$ at the $50^{\text {th }}$ cycle.

\section{CONCLUSION}

We have demonstrated a functional flexible lithium ion battery. Our process is generic for its utilization of conventional Si substrate, compatible with CMOS processes. The transfer approach of cells on polymer substrate before etching results in high yield production and ease of handling fragile flexible samples. Comparison between a flexible and rigid LIB showed comparable electrochemical performance. Future work will focus on the correlation between the mechanical strain on the nanostructure and microstructure of the LIB cathode material and its effect on the electrochemical performance. The LIB is promising for medical implantable applications in IoE and wearable electronics.

\section{REFERENCES}

[1] C. K. Jeong, K.-I. Park, J. H. Son, G.-T. Hwang, S. H. Lee, D. Y. Park, H. E. Lee, H. K. Lee, M. Byun, and K. J. Lee, "Self-powered fully-flexible light-emitting system enabled by flexible energy harvester," Energy Environ. Sci., vol. 7, pp. 4035-4043, 2014.

[2] L. Francioso, C. De Pascali, I. Farella, C. Martucci, Creti, x, P., P. Siciliano, and A. Perrone, "Flexible thermoelectric generator for wearable biometric sensors, " IEEE Sensors 2010, pp. 747-750, 2010

[3] G. A. T. Sevilla, S. B. Inayat, J. P. Rojas, A. M. Hussain, and M. M. Hussain, "Flexible and Semi-Transparent Thermoelectric Energy Harvesters from Low Cost Bulk Silicon (100),” Small, vol. 9, pp. 39163921, 2013.

[4] S. S. Shin, W. S. Yang, J. H. Noh, J. H. Suk, N. J. Jeon, J. H. Park, J. S. Kim, W. M. Seong, and S. I. Seok, "High-performance flexible perovskite solar cells exploiting $\mathrm{Zn}_{2} \mathrm{SnO}_{4}$ prepared in solution below $100{ }^{\circ} \mathrm{C}$," Nat Commun., vol. 6, 2015.

[5] M. T. Ghoneim, M. A. Zidan, M. Y. Alnassar, A. N. Hanna, J. Kosel, K. N. Salama, and M. M. Hussain, "Thin PZT-Based Ferroelectric Capacitors on Flexible Silicon for Nonvolatile Memory Applications," Adv. Electron. Mater., vol. 1, 2015.

[6] L. Liu, Y. Yu, C. Yan, K. Li, and Z. Zheng, "Wearable energy-dense and power-dense supercapacitor yarns enabled by scalable graphene-metallic textile composite electrodes,” Nat. Commun., vol. 6, 2015.

[7] M. F. El-Kady and R. B. Kaner, "Scalable fabrication of high-power graphene micro-supercapacitors for flexible and on-chip energy storage," Nat. Commun., 2013.

[8] L. Yuan, X.-H. Lu, X. Xiao, T. Zhai, J. Dai, F. Zhang, B. Hu, X. Wang, L. Gong, J. Chen, C. Hu, Y. Tong, J. Zhou, and Z. L. Wang, "Flexible Solid-State Supercapacitors Based on Carbon Nanoparticles/MnO2 Nanorods Hybrid Structure,” ACS Nano, vol. 6, pp. 656-661, 2012.

[9] Z. Song, T. Ma, R. Tang, Q. Cheng, X. Wang, D. Krishnaraju, R. Panat, C. K. Chan, H. Yu, and H. Jiang, "Origami lithium-ion batteries," Nat. Commun., vol. 5, 2014.

[10] Y.-H. Lee, J.-S. Kim, J. Noh, I. Lee, H. J. Kim, S. Choi, J. Seo, S. Jeon, T.-S. Kim, J.-Y. Lee, and J. W. Choi, "Wearable Textile Battery Rechargeable by Solar Energy,” Nano Lett., vol. 13, pp. 5753-5761, 2013.

[11] G. Zhou, F. Li, and H.-M. Cheng, "Progress in flexible lithium batteries and future prospects,” Energy Env. Sci., vol. 7, pp. 1307-1338, 2014.

[12] C. Yan and P. S. Lee, "Stretchable Energy Storage and Conversion Devices,” Small, vol. 10, pp. 3443-3460, 2014.

[13] K. Xie and B. Wei, "Materials and Structures for Stretchable Energy Storage and Conversion Devices,” Adv. Mater., vol. 26, pp. 3592-3617, 2014. 
[14] N. Aliahmad, M. Agarwal, S. Shrestha, and K. Varahramyan, "PaperBased Lithium-Ion Batteries Using Carbon Nanotube-Coated Wood Microfibers,” IEEE Trans. Nanotechnol., vol. 12, pp. 408-412, 2013.

[15] H. Cheng, C. Hu, Y. Zhao, and L. Qu, "Graphene fiber: a new material platform for unique applications," NPG Asia Mater., 2014

[16] L. Jabbour, C. Gerbaldi, D. Chaussy, E. Zeno, S. Bodoardo, and D. Beneventi, "Microfibrillated cellulose-graphite nanocomposites for highly flexible paper-like Li-ion battery electrodes,” J. Mater. Chem., vol. 20, pp. 7344-7347, 2010.

[17] B. Liu, J. Zhang, X. Wang, G. Chen, D. Chen, C. Zhou, and G. Shen, "Hierarchical Three-Dimensional $\mathrm{ZnCo}_{2} \mathrm{O}_{4}$ Nanowire Arrays/Carbon Cloth Anodes for a Novel Class of High-Performance Flexible LithiumIon Batteries," Nano Lett., vol. 12, pp. 3005-3011, 2012.

[18] J. B. Bates, N. J. Dudney, G. R. Gruzalski, R. A. Zuhr, A. Choudhury, C. F. Luck, and J. D. Robertson, "Electrical properties of amorphous lithium electrolyte thin films " Solid State Ionics, vol. 53-56, pp. 647-654, 1992.

[19] H. J. Ha, E. H. Kil,Y. H. Kwon,,J. Y. Kim,' C. K. Lee' and Sang-Y. Lee, "UV-curable semi-interpenetrating polymer network-integrated, highly bendable plastic crystal composite electrolytes for shape-conformable allsolid-state lithium ion batteries," Energy Environ. Sci., vol. 5, pp. 64916499, 2012.

[20] E. Quartarone and P. Mustarelli, "Electrolytes for solid-state lithium rechargeable batteries: recent advances and perspectives," Chem. Soc. Rev., vol. 40, pp. 2525-2540, 2011.

[21] L. Kai, S. Yinghui, Z. Ruifeng, Z. Hanyu, W. Jiaping, L. Liang, F. Shoushan, and J. Kaili, "Carbon nanotube yarns with high tensile strength made by a twisting and shrinking method," Nanotechnology, vol. 21, no. 4, pp. 045708-045714, 2010.

[22] L. Wen, F. Li, H.-Z. Luo, and H.-M. Cheng, "Graphene for Flexible Lithium-Ion Batteries: Development and Prospects" in Nanocarbons for Advanced Energy Storage, Volume 1 (ed X. Feng), Wiley-VCH Verlag GmbH \& Co. KGaA, Weinheim, Germany, 2015.

[23] X. Fang, C. Shen, M. Ge, J. Rong, Y. Liu, A. Zhang, F. Wei, and C. Zhou, "High-power lithium ion batteries based on flexible and light-weight cathode of LiNi0.5Mn1.5O4/carbon nanotube film, ” Nano Energy, vol. 12, pp. 43-51, 2015.

[24] J. P. Rojas, M. T. Ghoneim, C. D. Young, and M. M. Hussain, "Flexible High- $/$ /Metal Gate Metal/Insulator/Metal Capacitors on Silicon (100) Fabric," IEEE Trans. Electron. Dev., vol. 60, no. 10, p. 3305-3009, 2013.

[25] G. A. Torres Sevilla, M. T. Ghoneim, H. Fahad, J. P. Rojas, A. M. Hussain, and M. M. Hussain, "Flexible Nanoscale High-Performance FinFETs,” ACS Nano, vol. 8, pp. 9850-9856, 2014.

[26] X. Wang, X. Lu, B. Liu, D. Chen, Y. Tong, and G. Shen, "Flexible Energy-Storage Devices: Design Consideration and Recent Progress," Adv. Mater., vol. 26, pp. 4763-4782, 2014.

[27] C. Wang, W. Zheng, Z. Yue, C. O. Too, and G. G. Wallace, "Buckled, Stretchable Polypyrrole Electrodes for Battery Applications," Adv. Mater., vol. 23, pp. 3580-3584, 2011.

[28] S. Xu, Y. Zhang, J. Cho, J. Lee, X. Huang, L. Jia, J. A. Fan, Y. Su, J. Su, H. Zhang, H. Cheng, B. Lu, C. Yu, C. Chuang, T.-i. Kim, T. Song, K. Shigeta, S. Kang, C. Dagdeviren, I. Petrov, P. V. Braun, Y. Huang, U. Paik, and J. A. Rogers, "Stretchable batteries with self-similar serpentine interconnects and integrated wireless recharging systems," Nat. Commun., vol. 4, article no. 1543, 2013.

[29] M. Koo, K.-I. Park, S. H. Lee, M. Suh, D. Y. Jeon, J. W. Choi, K. Kang, and K. J. Lee, "Bendable Inorganic Thin-Film Battery for Fully Flexible Electronic Systems,” Nano Lett., vol. 12, pp. 4810-4816, 2012.

[30] J. B. Bates, N. J. Dudney, B. Neudecker, A. Ueda, and C. D. Evans, "Thinfilm lithium and lithium-ion batteries," Solid State Ionics, vol. 135, pp. 33-45, 2000.

[31] B. J. Neudecker, N. J. Dudney, and J. B. Bates, "Lithium-Free Thin-Film Battery with In Situ Plated Li Anode,” J. Electrochem. Soc., vol. 147, pp. 517-523, 2000.

[32] C. corporation. (2012 ). Cymbet EnerChip Bare Die Solid State Batteries Verified Non-cytotoxic Available:http://www.cymbet.com/products/ecofriendly-features.php

[33] F. Ning, S. Li, B. Xu, and C. Ouyang, "Strain tuned Li diffusion in LiCoO2 material for Li ion batteries: A first principles study,” Solid State Ionics, vol. 263, pp. 46-48, 2014.

Arwa T. Kutbee is currently a $\mathrm{PhD}$ candidate in the Materials Science and Engineering Program, KAUST. She is developing various free form energy storage devices.
Mohamed T. Ghoneim (S'10) is currently pursuing a $\mathrm{PhD}$ degree in the Electrical Engineering Program, KAUST. His current research interests include free-form memory devices and reliability physics of free-form electronics.

Sally M Ahmad: is a PhD student in the Electrical Engineering Program, KAUST. She works on modeling and simulation of free-form electronics.

Muhammad M. Hussain (M'07-SM'10) is an Associate Professor with the Electrical Engineering Program, KAUST. He works on unexplored new applications of electronics. 